This item was submitted to Loughborough's Research Repository by the author.

Items in Figshare are protected by copyright, with all rights reserved, unless otherwise indicated.

\title{
A novel, modular validation framework for collision avoidance of automated vehicles at road junctions
}

PLEASE CITE THE PUBLISHED VERSION

https://doi.org/10.1109/ITSC.2018.8569631

PUBLISHER

(C) IEEE

VERSION

AM (Accepted Manuscript)

LICENCE

CC BY-NC-ND 4.0

\section{REPOSITORY RECORD}

Nitsche, Philippe, Ruth Welsh, A. Genser, and Pete Thomas. 2018. "A Novel, Modular Validation Framework for Collision Avoidance of Automated Vehicles at Road Junctions". Loughborough University. https://hdl.handle.net/2134/37051. 


\title{
A novel, modular validation framework for collision avoidance of automated vehicles at road junctions*
}

\author{
P. Nitsche, R.H. Welsh, A. Genser and P.D. Thomas
}

\begin{abstract}
This paper presents a new validation method for automated driving systems at road junctions. The method comprises the clustering of critical traffic scenarios at junctions as well as a simulation and evaluation framework to validate those scenarios. The safety performance indicators selected and implemented in the framework can be seen as a new reference for conducting virtual tests at junctions. The applicability of the framework is demonstrated by an experiment based on a selected car-to-car collision scenario. Considering the current progression of automated transport, this work is highly relevant for virtual testing procedures and is an important step towards approval and certification of automated vehicles.
\end{abstract}

\section{INTRODUCTION}

For automated vehicles (AV), road junctions constitute a major point of interest along their routes due to the increased likelihood of conflicts with other road users. AV should be capable of safely manoeuvring through an intersection and of avoiding or mitigating a collision. Consequently, there is a need for comprehensive testing, either in virtual simulation environments or on real-world test tracks. The objective of this research is to provide a novel validation tool for testing the safety of AV in road junction environments. This tool is realised in the form of a simulation and evaluation framework, which leads to more efficient workflows in virtual tests. The work includes (a) a method to generate safety-critical testing scenarios from accident data, (b) a method to virtually reconstruct and evaluate the scenarios using stochastic parametric variations in a sub-microscopic simulation environment, and (c) the application of specific indicators to quantify the safety performance of an AV's collision avoidance (CA) system.

This paper is structured as follows: Background on related work and previous studies is presented in Section II, before Section III explains the developed validation framework in detail. The usefulness and applicability of the framework is demonstrated in Section IV by presenting the results from a simulation experiment for a selected junction scenario. A discussion about the applications and limitations is given in Section V, before Section VI concludes the paper with final remarks and an outlook on future work.

\footnotetext{
* P. Nitsche and A. Genser are with the AIT Austrian Institute of Technology, Center for Mobility Systems, Giefinggasse 2, 1210 Vienna, Austria (phone: +436626207865; fax: +43505506439; e-mails: philippe.nitsche@ait.ac.at, alexander.genser@ait.ac.at).

P.D. Thomas and R.H. Welsh are with Loughborough University, LE11 3TT, UK (e-mails: p.d.thomas@lboro.ac.uk, r.h.welsh@lboro.ac.uk)
}

\section{BACKGROUND AND MOTIVATION}

Automated driving can be seen as the evolutionary progress from advanced driver assistance systems (ADAS). Numerous research studies investigated the safety effectiveness of different ADAS functions, e.g. in the projects ASSESS [1], PReVAL [2], interacIVe [3] or in the U.S. [4][6]. However, the developed assessment frameworks cannot be directly applied to AV due to their more complex requirements [7], [8]. In comparison to ADAS, AV testing scenarios need to cover the complete driving process and possible situations, the automated driving function is expected to operate over a longer time and there might not be driver interaction anymore. Hence, the main challenge is to identify the key driving situations to be evaluated. The findings from [9] and [10] support the need for alternative testing methods to supplement real-world tests, in particular by virtual tests and vehicle simulations. Virtual validation procedures for AV have been developed in recent projects such as adaptIVe [11], PEGASUS [12], [13], ENABLE-S3 [14] or ADAS\&ME [15]. However, there is little research on testing scenarios for road junction areas. Every third accident occurs at a junction, which can therefore be considered as a relevant risk area [16]. Despite the many projects and research studies in the field of intersection CA (e.g. [17][20]), there is still no commercially available intersection assistant system on the market, which can cope with all intersection types and possible situations. One reason for this could be the lack of common testing procedures for junction situations, which is the focus of this paper.

This work provides three novel contributions to existing approaches. First, the junction scenarios to be evaluated are obtained from accident data by using a combination of clustering and association rule mining, as published by Nitsche et al. [21]. A database-driven method to follow this holistic approach was developed by Pütz et al. [12]. This paper focuses on one aspect of their knowledge base, namely the analysis of accident data to identify critical testing. Most publications on intersection scenarios focused on the classification of pre-crash manoeuvres, not combined with parameters about the road environment, collision partners, points of impact, injury types, causation factors and traffic control. Kim et al. [22] identified sixteen vehicle-to-vehicle accident scenarios at intersections and studied the accident prevention capabilities of camera-based and radar-based collision warning in those scenarios. The study primarily focused on vehicle movements and did therefore not differentiate between different junction types and did not include information about traffic control or speed limits. 
Compared to literature, the work presented in this paper can be seen as more detailed in terms of crash circumstances.

The second novelty is that the junction simulations are based on the Latin Hybercube Sampling (LHS) method [23], which divides the cumulative probability curve into equal intervals on the scale and samples one random value from each interval of the input distribution. Available studies, e.g. [24], [25], applied the traditional Monte Carlo method, which has the disadvantage that more runs, and hence more computational effort, may be necessary to obtain representative results. In general, the approach presented in this paper is different from traditional crash simulation studies, where real-world road locations are reconstructed case by case from a crash sample of the underlying accident database and the effectiveness or crash avoidance rates of safety interventions are studied [26]-[30]. Commonly, those studies use a crash reconstruction tool such as the software PC-Crash. In this research, the virtual junction is not necessarily included in the underlying crash data samples, because the samples are not evaluated case by case. Instead, functional abstract scenarios are obtained from clustering and further specified by association rules. Concrete, less abstract scenarios are then produced by parametric variation using the LHS sampling method (see Table I). In other words, artificial critical scenarios are evaluated instead of accident scenarios that really happened. In this way, more parametric variation can be implied than by using a limited crash population.

TABLE I. TYPES OF SCENARIOS AND HOW THEY ARE DERIVED

\begin{tabular}{|l|c|c|c|}
\hline \multicolumn{1}{|c|}{ Type: } & Functional scenario & Logical scenario & Concrete scenario \\
\hline $\begin{array}{l}\text { Level of } \\
\text { abstraction: }\end{array}$ & High & $\rightarrow$ & Low \\
\hline $\begin{array}{l}\text { Obtained } \\
\text { by: }\end{array}$ & Clustering results & $\begin{array}{c}\text { Association rule } \\
\text { results }\end{array}$ & $\begin{array}{c}\text { Parametric variation } \\
\text { (LHS) }\end{array}$ \\
\hline $\begin{array}{l}\text { Described } \\
\text { by: }\end{array}$ & Natural language & $\begin{array}{c}\text { Parameter ranges } \\
\text { and attributes }\end{array}$ & $\begin{array}{c}\text { Precise parameter } \\
\text { values }\end{array}$ \\
\hline
\end{tabular}

As the third novelty, a systematic procedure using a new concept called "conflict hexagon" along with a combination of safety metrics is provided to evaluate the performance of $\mathrm{AV}$ at junctions. The assessment of testing scenarios is commonly based on safety indicators, which can be used to measure the spatial and temporal proximity of safety-critical events, assuming that they have an established relationship with accidents [31]. Hence, the safety evaluation of simulation runs should not only result in collision probabilities, but also in near-miss probabilities. There has been significant research on appropriate indicators to evaluate the severity of road conflicts [31]-[36], but only a few studies have investigated indicators for simulated junction scenarios in particular. In [22], collision warning systems for intersections were evaluated based on scenarios derived from naturalistic driving data and accident records. However, their study focused on accident prevention rates and considered only one safety indicator and no conflicts. Since profound safety indicators are the most important elements of a virtual testing procedure, there is a need to establish a reference method to assess the safety performance of AVs and their CA capability at junctions.

\section{VALIDATION FRAMEWORK}

A new validation method was developed to address the abovementioned challenges. The work focusses on three- and four-legged at-grade junctions, either signalised or unsignalised. Fig. 1 depicts the overall framework, whose modules are further described in the following subsections. In general, the idea is to initially partition historical crash data (involving human drivers) by a clustering technique and then apply the association rule method on the data subsets (lefthand side of Fig. 1), inspired by [37]. The derived scenarios are then transferred to a sub-microscopic simulation environment for examining the safety performance (righthand side of Fig. 1) based on a combination of numerical indicators. The framework is set up according to one or more experiments to test pre-defined hypotheses related to the objectives of the virtual test. It replaces the driver with an $\mathrm{AV}$, further denoted as ego car. On the one hand, this affects the parameters for the ego car model, such as reaction times or driving behaviour. On the other hand, factors that are assumed to be problematic for AV must be taken into account. Risks that could negatively influence the safety performance of AV are specified as "criticalities". Hence, criticality factors are added to the simulation parameters derived from the association rule analysis.

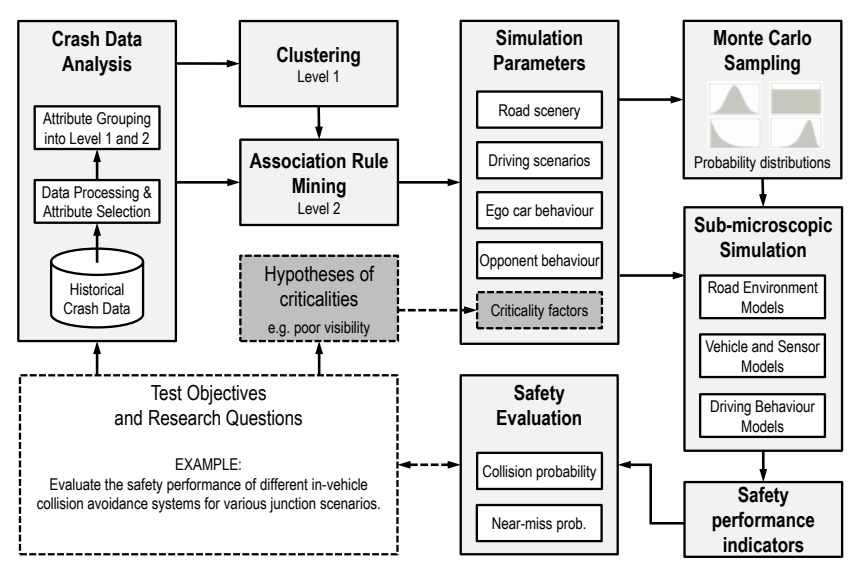

Figure 1. Validation framework

The overall framework is designed in a modular manner by providing particular methods for each of the modules. Depending on the objectives of the validation, not all of the modules must be applied, or particular methods can be replaced by others.

\section{A. Crash Data Analysis, Clustering and Association Rule Mining}

This section briefly describes the accident data mining approach. For a more detailed explanation of crash data attributes and results of the method, refer to [21], where this 2-step approach was applied to an in-depth junction accident dataset. Basically, the aim of the crash data analysis and mining is to identify distinct groups of safety-critical scenarios relevant for the test. Therefore, the dataset is divided into two levels. Level 1 is a reduced set of attributes describing the collision parameters, for better partitioning and easier interpretation of the results, while Level 2 adds additional attributes describing the environment and 
causation factors. Level-1 data are used as input for the clustering algorithm and level-2 data for finding association rules. This two-level approach was chosen (a) because most clustering methods achieve superior clustering results on a smaller set of attributes and no clear partitioning would be achieved when using all available attributes, and (b) because the results from applying the association rules on the whole dataset (without prior clustering) would be hard to interpret due to the high number of obtained rules.

After investigating different clustering techniques, the $k$ medoids method, in particular an algorithm called PAM (Partitioning Around Medians) [38], was applied to compute the clusters. $k$-medoids was found to be most appropriate for an accident dataset, since the method is robust against outliers and can cope with categorical data. The Hamming distance was used as similarity measure, since the input data is coded in a binary format. In an iterative process, the number of $k$ was incremented from 2 to 15 and the best $k$ was determined by taking into account the so-called average Silhouette value [39], the size of the clusters and the number of samples allocated to the wrong cluster.

Association rules are a popular method in data mining to discover hidden associations within variables in large datasets. In the presented framework, the Apriori algorithm [40] was applied, with a minimum confidence value of 0.75 and a minimum support value of 0.03 . Additionally, only rules with a lift value higher than 1.25 were taken to derive various logical crash scenarios, which can be described by four groups of simulation parameters: Road configuration, driving scenarios, ego car behaviour and opponent behaviour. Those parameters can then be modelled in the simulation environment.

\section{B. Simulation Parameter Sampling}

The scenarios obtained help to reduce the possible number of parameter variations for the simulation models, such as vehicle manoeuvres, trajectories, velocities as well as road and junction parameters. However, not all parameters for the simulations can be derived from the crash data analysis. Some data elements are simply not included in the accident database, such as exact driving trajectories or driving behaviour. The proposed methodology fills this gap by enhancing the collision scenarios with representative variations of real-world conditions, sampled by the Monte Carlo approach. The aim is to process a multidimensional input space consisting of varying parameters, each of which having a certain probability distribution. The basic tasks of the framework are (1) to generate random samples from the given input distributions, (2) to run simulations for each set of the samples and (3) to perform statistical analyses on the simulation output values.

The Monte Carlo method is usually applied to computationally inexpensive models, because there are a high number of samples necessary to obtain satisfactory results. The approach results in much lower confidence bounds when the sample size is reduced. To cope with this problem, the framework uses LHS, as explained in Section II. It takes even unlikely extremities into account as it is desired for vehicle tests. In the car-to-car demonstration experiment explained in Section IV, LHS sampling is applied to a three-dimensional input space (see Table IV). The sampling approach ensures that the input probability distributions are maintained and that the resulting simulation outputs are considered representative in this respect. By varying those three inputs, the ego car is exposed to a large amount of different situations that combined will lead to conclusions about the collision risk and near-miss risk for the system under test. Of course, the number of variations can be increased depending on the goal of the experiment, e.g. by adding a variation in driving style or vehicle and sensor parameters. However, note that the parametric variation for each experiment is constrained by the underlying logical scenario (see Table I), i.e. a variation of the overall collision parameters and driving manoeuvres would require a new experiment.

\section{Sub-microscopic Simulation}

The sampled simulation parameters are transferred to a sub-microscopic simulation environment for examining the safety performance of $\mathrm{AV}$ at junctions. In the demonstration experiment explained in Section IV, this was realized by using the computer simulation tool CarMaker in combination with MATLAB/Simulink. However, the simulation software can be replaced by any other tool that provides physical models, e.g. for tires, suspension, engine or sensors, and that has the capability to simulate their interaction with the surrounding road area.

A simulation experiment may contain various sceneries, i.e. road and junction environments to be analyzed. Each scenery itself contains one or more scenarios, derived from crash data. The original crash scenario is modified and simulated until the point of impact, by implementing three model groups: (1) Road environment, (2) vehicle and sensors and (3) driving behavior of the ego car and other road users. The method can therefore also be called a pre-crash simulation, because it does not replicate post-collision damage, injury and vehicle positions. The sensor models are used to replicate an AV's ability to sense its environments and also replicate their limitations due to poor visibility or sensor failure. The vehicle control module that perceives the sensor is specified by the driving behavior model, including the CA models.

In the developed simulation framework, the input for the simulations, i.e. the varying parameters and combinations of those are specified by an XML-structured configuration file. An automation script is implemented in MATLAB and sets up the simulation environment, interprets the input XML-file and transfers the input parameters to the Simulink instance, where the values are assigned to CarMaker model blocks for initializing the simulations. The CarMaker GUI is then executed to perform the simulation runs and to deliver the specified output quantities for each run. Those output files are stored in the simulation database and are post-processed to compute the safety indicators, which are described in the following subsection. Finally, the resulting indicators, including the information if it was a collision, a conflict or an undisturbed situation are stored in the results database.

For an intersection situation, an AV must be able to predict potential collisions for a variety of scenarios, such as head-on, rear-end, turning or right-angle collisions. This simulation and evaluation framework uses two collision avoidance models as part of the driving behavior models, 
which were developed in Simulink, and which are compared to each other in the demonstration experiment in Section IV:

1. Forward collision avoidance (FCA), based on forward-facing vehicle sensors, also known as automated emergency brake, to sense other road users in front of the vehicle and to avoid rear-end and head-on collisions by braking actions. At the moment, steering evasion actions are disregarded.

2. Crossing and turning assistance (CTA), based on both forward and sideward-looking sensors, designed to find a safe gap to cross or turn and to avoid side and angle collisions (see Fig. 3). The model uses the metrics Time-ToIntersection (TTI), Time-To-Disappear (TTD), Time-ToReact $(T T R)$ and Gap Acceptance (GA) (see Tbl. II) and decides on the driving action according to Eq. 1. Note that $T T I$ and TTD are continuous values changing over time $t$, while TTR and GA are constant values.

$$
f(t)= \begin{cases}T u r n, & \text { if } T T I_{2}(t)-T T D_{1}(t)-T T R_{1}>G A \\ \text { Wait, } & \text { if } T_{T I}(t)-T T D_{1}(t)-T T R_{1} \leq G A\end{cases}
$$

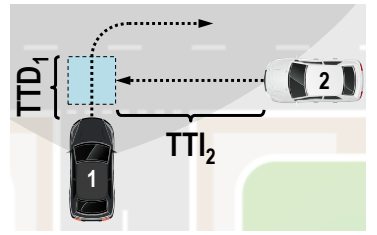

Figure 2. Illustration of the crossing and turning assistant model

\section{Safety Performance Indicators and Evaluation}

The previous section described the overall simulation module design and the CA models. Each simulation run has one output file including all defined quantities, which is further analyzed by an evaluation script to derive safety indicators. This section is devoted to the metrics used to quantify the safety performance of the $\mathrm{AV}$ in the given scenario.

As Table II shows, there are three uses of indicators in the framework, namely (a) metrics used for the apriori CA algorithm that are computed during the simulation runtime (see previous section), (b) metrics used for the a-posteriori detection and severity estimation of collisions and (c) metrics used for the a-posteriori detection of conflicts or near-misses. While the apriori indicators are the basis for the CA system of the ego car, the a-posteriori indicators are computed after each simulation run as safety performance metrics. The evaluation script detects whether there was a collision or a conflict, in order to compute a conflict probability along with a collision probability. Furthermore, the collision injury severity is estimated.

The choice of a posteriori indicators depends on the collision scenario to be evaluated. The PET is defined as the time difference between one road user exiting a spatial area and another road user entering it. It is used for angle, side and turning collision scenarios, where both road users' trajectories do not end in the same path and direction, but it is not applicable to rear-end, merging or head-on situations. For those scenarios, the minimum TTC or its derivatives such as the time-integrated TTC are preferred. The $T T C$ is further applied to the cases where an evasion action, e.g. a braking maneuver, was taken by either of the road users. From the timestamp where a critical braking maneuver was detected, the $T T C$ is computed a posteriori. In literature, this application of $T T C$ is also denoted Time-To-Accident (TTA) [41]. Accordingly, the TTA is defined as "the time that remains to an accident from the moment that one of the road users starts an evasive action if they had continued with unchanged speeds and directions" [42]. The TTA can be related to the conflict speed in order to define conflict severity levels [41]. The conflict speed is defined as the vehicle's velocity at the moment the evasion action is taken. In comparison to the traditional approach with a fixed TTC/TTA threshold, this threshold function has the advantage that the conflict severity is speed-dependent and hence more reliable.

TABLE II. SAFETY PERFORMANCE INDICATORS USED

\begin{tabular}{|l|l|c|}
\hline \multicolumn{1}{|c|}{ Use } & Safety indicator name & (Threshold) Value \\
\hline \multirow{3}{*}{$\begin{array}{l}\text { Collision } \\
\text { avoidance } \\
\text { a priori) }\end{array}$} & Time-To-Intersection $(T T I)$ & Continuous value \\
\cline { 2 - 3 } & Time-To-Disappear $(T T D)$ & Continuous value \\
\cline { 2 - 3 } & Time-To-React $(T T R)$ & $0.01 \mathrm{~s}$ \\
\cline { 2 - 3 } $\begin{array}{l}\text { Collision detection } \\
\text { and severity } \\
\text { estimation } \\
\text { (a posteriori) }\end{array}$ & Gap acceptance time $(G A)$ & $1 \mathrm{~s}$ \\
\cline { 2 - 3 } $\begin{array}{l}\text { Conflict detection } \\
\text { (a posteriori) }\end{array}$ & Collision detection & $0 / 1$ \\
\cline { 2 - 3 } & Impact angle $(\varnothing)$ & $\mathrm{n} / \mathrm{a}$ \\
\cline { 2 - 3 } & Delta of velocity $(D V)$ & $\mathrm{n} / \mathrm{a}$ \\
\cline { 2 - 3 } & Time-To-Collision $(T T C)$ & Threshold: $<1.5 \mathrm{~s}$ \\
\cline { 2 - 3 } & Pime-To-Accident $(T T A)$ & Threshold function \\
\cline { 2 - 3 } & Lost Encroachment Time $(P E T)$ & Threshold: $<1 \mathrm{~s}$ \\
\hline
\end{tabular}

Fig. 3 depicts a flowchart of the evaluation procedure as realized in the framework. When a collision is detected, the aim is to estimate the severity in terms of injury risk. To do this, the impact velocities and the impact angle are calculated to obtain the Delta of Velocity $(D V)$, along with the information which vehicle is the 'bullet' vehicle. The $D V$ is calculated by the following approximation based on Newton's mechanics of momentum conservation:

$$
D V_{1}=\frac{m_{2}}{m_{1}+m_{2}} \sqrt{I V_{1}^{2}+I V_{2}^{2}-2 I V_{1} I V_{2} \cos \emptyset}
$$

with $m_{1}$ and $m_{2}$ as the masses of vehicle 1 and vehicle 2, respectively, $I V_{1}$ and $I V_{2}$ as their impact speeds and $\emptyset$ as the included angle between the axis of travel of both vehicles. Note that if vehicle 2 collides with vehicle 1 at a greater angle than 90 degrees, then $I V_{2}$ becomes negative in Eq. 2 . It is significant to mention that this approximation is simplified, as other important factors such as the part of the vehicles hit, the vehicles' stiffness, the vehicles' rotation or the postimpact rebound are not included, i.e. that the collision is assumed inelastic.

In [43], crash data from 1995 to 1999 were examined to derive a relationship between $D V$ and the probability of occupant injury along all different MAIS (Maximum Abbreviated Injury Scale) levels, as a basis for estimating the impact of improved stopping distance on vehicle safety. The percent probability risk of each MAIS level at each $D V$ was defined as the number of MAIS injuries divided by the total number of occupants involved at a certain $D V$. The risk prediction curves were derived using a mathematical 
modelling process with $D V$ as the independent variable and the probability risk as the dependent variable. The presented framework uses those risk function to estimate the most likely injury severity level. However, the formulas have to be used with care, because they might not be applicable to all types of collisions. Furthermore, the study included only accidents, where at least one vehicle used the brakes.

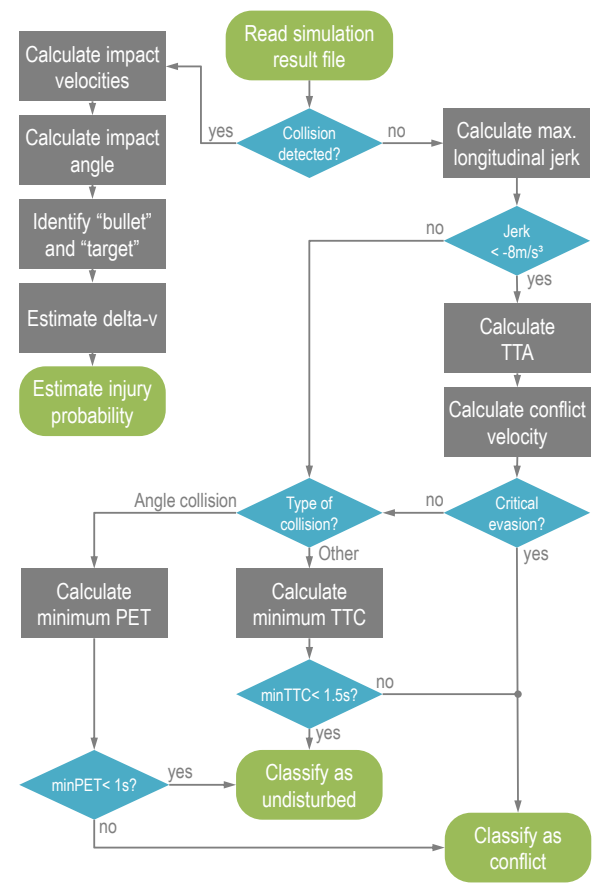

Figure 3. A posteriori evaluation of the safety performance

In case there is no collision detected, the aim is to find out whether the scenario is a critical conflict or an undisturbed situation. There are two decision points to answer this: First, did one of the vehicles perform an evasion maneuver, i.e. an emergency braking? Second, did the vehicles come close to each other, even if there was no braking involved. The first question is answered by the maximum jerk value. If it exceeds the threshold, the TTA-conflict-velocity function in [41] indicates if the braking maneuver was performed in a critical conflict situation. The PET or the TTC thresholds give an indication on the second question, depending on whether there was an angle (side) collision or another collision type. Computing the TTC for crossing path or turning against situations is not trivial, since the trajectories of the road users have to be predicted in order to find a conflict point. This means that a real-world implementation is complex and suffers prediction inaccuracy. Previous studies used a conflict point as a reference for the calculation of the TTC and the PET between two crossing road users, e.g. [44]. This framework instead introduces a conflict hexagon, which advances the conflict zone principle published in [34], [45], where the zone is spanned by the vehicle dimensions, with the vehicles represented as rectangular objects. For the case of precisely rectangular collisions, the form of the conflict zone is a rectangle. However, with changing angles, the conflict zone becomes a hexagon, as illustrated in Fig. 4. Consequently, each vehicle has its own conflict entry and exit points, which are used to compute TTC and PET. If both vehicles were driving within the hexagon, a collision would be imminent.
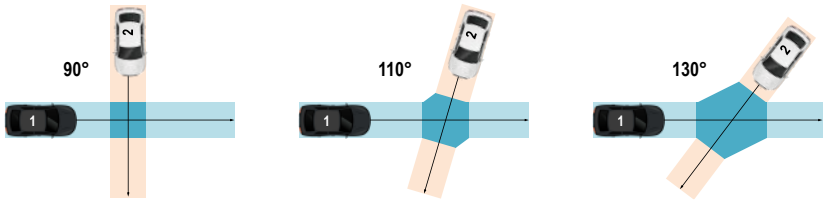

Figure 4. Different shapes of the conflict hexagon depending on the collision angle

\section{DEMONSTRATION EXPERIMENT}

The advantage of the proposed framework is that its modular architecture allows a number of different test objectives, e.g. to compare different vehicle sensor systems, evaluate varying intersection designs or assess the impacts of different driving behavior models. This section presents the results of a demonstration experiment to showcase the level of usefulness and versatility.

\section{A. Test scenario, road and vehicle models}

For the demonstration, it was chosen to focus on the comparison of two different in-vehicle sensor systems with respect to their crash avoidance capabilities. In particular, a right-turn manoeuvre by an AV at an unsignalised T-junction was evaluated, where another car is crossing from the right with the right of way. It was decided to virtually reconstruct a rural road junction with $40 \mathrm{mph}$ speed limit (i.e. $17.7 \mathrm{~m} / \mathrm{s}$ ) and left-hand traffic, located in the East Midlands, UK (see Fig. 5). It is a simple T-junction (without any traffic islands or dualling features), with a minor road terminated by a major road. It can be seen that the overall sight distance is limited, the junction angle is not orthogonal, both paths have one lane in each direction with relatively narrow widths, and the yield instructions are indicated by a give-way sign and a road marking before the stop line.

The ego car was simulated as detailed physical AV model, which requires a number of model parameter assumptions, such as vehicle dimensions, weight, engine, transmission, suspension or sensors. Therefore, the electric vehicle Tesla Model S was taken as basis for the car model. A combination of three video cameras was assumed $(1 \mathrm{x}$ forward and 2x sidewards), which were modelled as object sensors able to detect objects and other road users within their range. Those object sensors were the primary source for the recognition of other road users and the CA models.
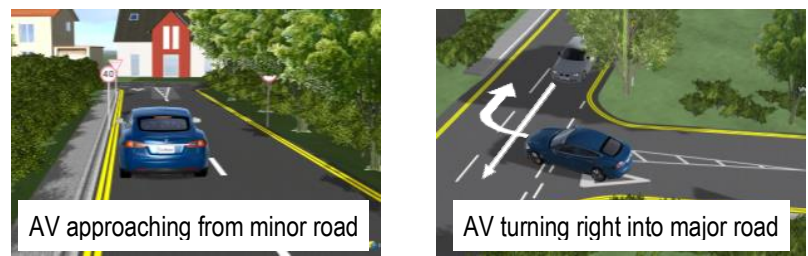

Figure 5. T-junction used for the demonstration

The opponent vehicle (OV) model is simplified and does not include physical dynamics models and sensor models. It is modelled as a so-called traffic object that can react to other traffic objects or the ego car, e.g. to avoid collisions or to follow behind. Vice versa, the ego car is able to detect the traffic object by its sensors. 


\section{B. Parameter variations}

Given the test objectives, the main parameter to vary for the comparison is the type of CA system of the ego car (see Table III). Since the CA models are modelled as ideal systems, i.e. they do not have any faults, a fault was injected that leads to a system failure. This failure is represented by two different object detection probabilities, i.e. the likelihood that another road user is detected by the vehicle sensors within their range, where $95 \%$ defines a rather satisfying value and $75 \%$ a rather poor value. This assumption is simplified, since in reality the detection probability depends on several influencing parameters such as light conditions or weather and varies over time. In the presented case, the detection state was either 0 or 1 for each run, meaning that from 100 runs the opponent vehicle will not be detected in 5 runs or 25 runs, depending on the probability variation. Those criticalities or "detection failures" were distributed randomly among all runs.

TABLE III. VARYING PARAMETERS WITH TWO STATES EACH TO COMPARE AGAINST

\begin{tabular}{|l|c|}
\hline Parameter name & Possible states \\
\hline Collision avoidance system & {$[$ FCA, CTA $]$} \\
\hline Object detection probability by vehicle sensor & {$[95 \%, 75 \%]$} \\
\hline
\end{tabular}

TABLE IV. VARYING PARAMETERS FOR THE LHS SAMPLING

\begin{tabular}{|l|c|c|c|c|}
\hline Parameter name & Range & Distrib. & Mean & Std. dev. \\
\hline $\begin{array}{l}\text { Opponent's lateral } \\
\text { position in lane }\end{array}$ & {$[-1 ; 1] \mathrm{m}$} & Gaussian & $\begin{array}{c}0 \mathrm{~m} \\
(\text { centre })\end{array}$ & $0.2 \mathrm{~m}$ \\
\hline $\begin{array}{l}\text { Opponent's } \\
\text { approaching velocity }\end{array}$ & {$[0 ; 17.7] \mathrm{m} / \mathrm{s}$} & Gaussian & $17 \mathrm{~m} / \mathrm{s}$ & $4 \mathrm{~m} / \mathrm{s}$ \\
\hline $\begin{array}{l}\text { Surface friction } \\
\text { coefficient }\end{array}$ & {$[0.3 ; 0.9]$} & Uniform & $\mathrm{n} / \mathrm{a}$ & $\mathrm{n} / \mathrm{a}$ \\
\hline
\end{tabular}

In addition, three LHS parameters (see Table IV) were sampled from predefined probability distributions, as explained in Section III.B. These included the opponent's lateral position within the lane and its velocity when approaching the junction as well as the surface friction coefficient, which influences the braking distance and vehicle stability. While the first two parameters were sampled from a normal distribution, the latter one was based on a uniform distribution in the range from 0.3 to 0.9 . The output dataset (from the LHS) for comparison was set to 4,000 samples for each of the four variable combinations, respectively. This resulted in 16,000 samples, i.e. simulation scenarios, which comprised around 142 hours of driving for the ego car. Considering that each parameter combination requires a reference run to obtain the theoretical, ideal vehicle trajectories, the number of simulation result files increased to 20,000 .

\section{Example evaluation of a collision}

The following example simulation run was picked from the collisions that resulted when using the FCA system with 75 percent detection probability. It is shown to demonstrate the evaluation procedure based on the collision indicators. Fig. 6 plots the trajectories within the global coordinate system of the simulation environment. Note that for the ego car, denoted as A, there are two trajectories depicted, namely the front axle centre plus/minus half of the vehicle width (blue lines) as well as the rear axle centre plus/minus half of the vehicle width (grey lines). The ochre lines represent the opponent car B's trajectory. Since A was turning, its tractrix curve must be taken into account to calculate the size of the conflict hexagon. The green dots indicate the hexagon entry points of both vehicles and the resulting hexagon is highlighted in red. The red dots represent the cars' position at the time of the collision. In CarMaker, the origin of the ego vehicle frame is located at the rear of the vehicle. To calculate the hexagon entry point precisely, the front most point of the ego car is taken as a reference point in the global coordinate system. This is done in CarMaker by placing a socalled inertial sensor on the vehicle front and to output its coordinates. The opponent car model is simplified and there is no option to place an inertial sensor. Hence, for car B the reference point remains at the rear of the vehicle, which is why the red dot is located there. This means that the distance to the hexagon entry point had to be corrected by the vehicle length. For the exit points, the time at which the vehicles pass with their rear end is relevant.

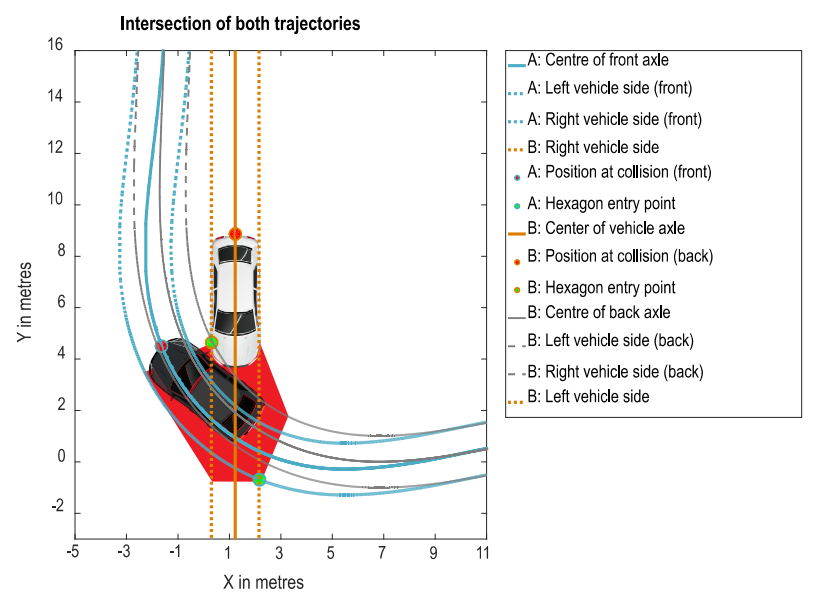

Figure 6. Trajectory plot (right) of a collision, with the AV turning right coming from the west, and the opponent car driving southwards

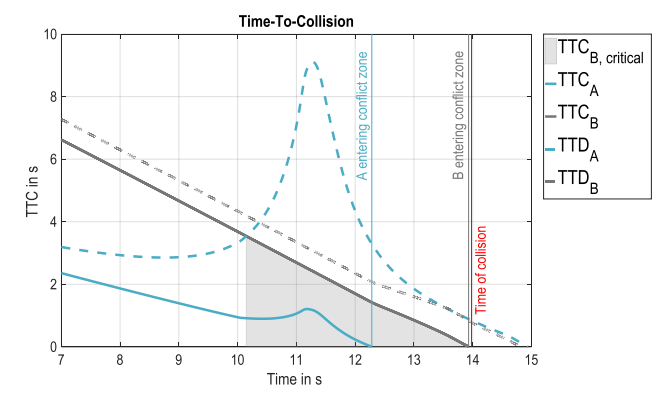

Figure 7. TTC and TTD over time for both vehicles in a collision scenario

The TTC curve is shown in Fig. 7. The TTC and TTD of the ego car is coloured in blue, while the curves of the opponent are shown in ochre. Three timestamps are plotted by vertical lines, namely the time of collision and the time when the vehicles entered the conflict zone. TTC and TTD are continuous values computed at each timestamp and decrease as the vehicles approach the conflict zone. The increase of TTC and TTD of the ego car after 10 seconds can be explained by the stop before turning into the junction and therefore decreased velocity. The TTC values become zero (and theoretically negative after that) at the time when the conflict zone is reached. The grey area reveals the timespan 
at which a critical $T T C$ is reached. The example in Fig. 8 shows that as long as car A has not left the conflict zone, i.e. as long as $T T D_{A}>T T C_{B}$, car $\mathrm{B}$ is on theoretical collision course, assuming that both vehicles continue driving at the same speed.

\section{Results of the safety evaluation}

The presented framework provides a large number of evaluation results and charts to analyse the safety performance. For brevity, only a few results are depicted, namely the overall results on the number of collisions and conflicts (Table V) and the influence of the LHS variation on injury probability (Fig. 8).

TABLE V. RESULTED PROBABILITIES FOR A COLLISION, CONFLICT AND UNDISTURBED SITUATION FOR ALL PARAMETER COMBINATIONS

\begin{tabular}{|l|c|c|c|}
\hline Parameter combinations & Collision & Conflict & Undisturbed \\
\hline FCA, 75\% detection probability & $30.3 \%$ & $9.5 \%$ & $60.2 \%$ \\
\hline FCA, 95\% detection probability & $29.9 \%$ & $9.3 \%$ & $60.9 \%$ \\
\hline CTA, 75\% detection probability & $7.6 \%$ & $4.2 \%$ & $88.2 \%$ \\
\hline CTA, 95\% detection probability & $1.2 \%$ & $2.3 \%$ & $96.5 \%$ \\
\hline
\end{tabular}

The safety performance evaluation resulted in 1,214 collisions and 379 conflicts for the worst-case setting compared to 49 collisions and 92 conflicts for the best case. For 95 percent detection probability, the number of collisions and conflicts were reduced to a tenth, when adding a CTA to the vehicle's CA system. This demonstrated the safety performance increase in this particular junction scenario. By using additional sideward-facing sensors with 95 percent detection probability, 96.5 percent of all simulations resulted in no collision or conflict.

A detailed analysis of the resulted collisions revealed that the injury probability strongly decreases from slight (mean value of $53 \%$ ) to serious (mean value of $2.3 \%$ ). The main reason for this can be found in the relatively low impact speeds of the ego car and the resulting low delta of velocity, which is used to estimate the injury severity. Apart from the fact that the frequencies strongly declined, it was found that the different sensor types and detection probabilities do not necessarily influence the distribution of injury probability. Instead, the varying Monte Carlo parameters do. For example, the fitted probability curve in Fig. 8 shows that a higher opponent velocity leads to a higher probability to suffer injury and that there is a slight increase of injury probability with a lower friction coefficient.

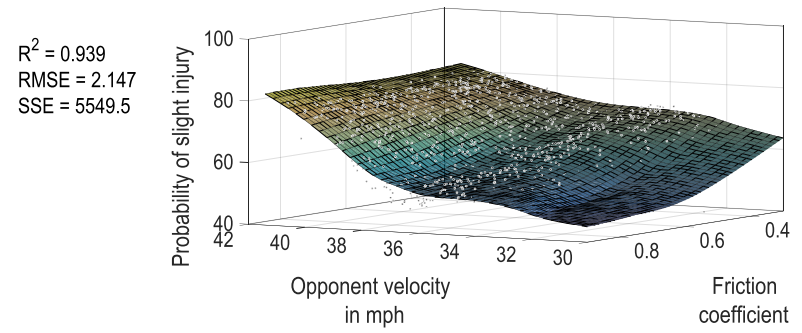

Figure 8. Fitted probability curve for slight injury by varying friction and opponent velocity, for collisions that occurred with FCA and $95 \%$ detection

The conflict indicator analysis also confirmed the hypothesis that a FCA system is not sufficient to avoid collisions with crossing traffic and therefore needs additional side collision assistance. Simulations with FCA only resulted in more critical braking situations than with additional CTA. In general, the additional sideward-facing sensor decreases the number of safety-critical events for both vehicles.

\section{CONCLUSION}

The presented research is the first work, to the author's knowledge, which thoroughly generated critical situations for testing automated driving at junctions, and which developed a simulation and evaluation framework to allow safety performance assessments for those junction scenarios. The framework was designed in a modular architecture, which can be adapted to the individual needs of future users and enhanced with customised MIL, SIL or HIL simulations. For example, the Simulink model blocks can be replaced by real hardware components or individual parameter combinations may be tested. This leads to more efficient workflows when virtually testing automated driving systems. Due to the principle of parametric variation and scenario generation, AV can be exposed to a large number of critical situations and their safety performance can be optimised towards better detection probability and crash avoidance capability. The applicability of the framework was demonstrated by an experiment based on a selected car-to-car collision scenario. It could be shown that the number of collisions and conflicts were reduced to a tenth when adding a CTA to the vehicle's FCA system. The safety performance indicators selected and implemented in the framework can be seen as a new reference for conducting virtual tests at junctions.

Limitations of the work include the simplified, inelastic computations of impacts and the fact that only primary collisions are considered. At the moment, only the severity of collisions is included and the information whether an evasion was critical or not. An interesting enhancement would be to include the severity of conflicts as well, since this would reveal more detailed insights into the effects of a varying parametrization of the vehicle, e.g. passive vs. sportive driving style. Another possible area of future research would be to investigate confidence rates of the given simulation results. For example, this could include information about the accuracy of the collision probability or injury severity, which would require a specification of the uncertainties in the applied models or, in the simple case, assuming a Poisson distribution for the rates. Furthermore, the computation efficiency is subject to future work to increase the number of simulation runs and parameter combinations.

\section{ACKNOWLEDGMENT}

The accident data in this study were acquired in cooperation with the UK Department for Transport that provided access to the RAIDS database.

\section{REFERENCES}

[1] A. Balint, H. Fagerlind, J.-A. Bühne, A. Aparicio, and E. McCarthy, "Safety impact assessment of integrated vehicle safety systems," ASSESS Deliverable D1.4, 2012.

[2] J. Scholliers et al., "Project final report and recommendations for future assessments," VTT Technical Research Centre of Finland, PReVAL Deliverable 16.4, Jan. 2008. 
[3] G. Alessandretti, A. Amditis, S. Metzner, E. Johannson, and F. Fahrenkrog, "interactIVe Final Report," interactIVe Deliverable D1.9, 2014.

[4] K. D. Kusano and H. C. Gabler, "Safety Benefits of Forward Collision Warning, Brake Assist, and Autonomous Braking Systems in Rear-End Collisions," IEEE Transactions on Intelligent Transportation Systems, vol. 13, no. 4, pp. 1546-1555, Dec. 2012.

[5] S. B. McLaughlin, J. M. Hankey, and T. A. Dingus, "A method for evaluating collision avoidance systems using naturalistic driving data," Accident Analysis \& Prevention, vol. 40, no. 1, pp. 8-16, Jan. 2008.

[6] S. B. McLaughlin, J. M. Hankey, T. A. Dingus, and S. E. Klauer, "Development of an FCW Algorithm Evaluation Methodology With Evaluation of Three Alert Algorithms: Final Report," NHTSA Report DOT HS 811 145, Jun. 2009.

[7] A. Zlocki, F. Fahrenkrog, M. Benmimoun, J. Josten, and L. Eckstein, "Evaluation of Automated Road Vehicles," in Road Vehicle Automation, Springer, Cham, 2014, pp. 197-208.

[8] C. Roesener et al., "A Comprehensive Evaluation Approach for Highly Automated Driving," in 25th International Technical Conference on the Enhanced Safety of Vehicles (ESV), 2017.

[9] N. Kalra and S. M. Paddock, "Driving to safety: How many miles of driving would it take to demonstrate autonomous vehicle reliability?," Transportation Research Part A: Policy and Practice, vol. 94, pp. 182-193, Dec. 2016.

[10] H. Winner and W. Wachenfeld, "Absicherung automatischen Fahrens," presented at the 6. Tagung Fahrassistenz, Munich, Germany, 2013.

[11] A. Etemad, "adaptIVe Final project results," adaptIVe Deliverable D1.0, Jun. 2017.

[12] A. Pütz, A. Zlocki, J. Bock, and L. Eckstein, "System validation of highly automated vehicles with a database of relevant traffic scenarios," in Proceedings of the 12th ITS European Congress, Strasbourg, France, 2017.

[13] K. Lemmer, "PEGASUS - Effectively ensuring automated driving," presented at the 19. VDA Technischer Kongress 2017, Berlin, Germany, 2017.

[14] H. Beglerovic, A. Ravi, N. Wikström, H.-M. Koegeler, A. Leitner, and J. Holzinger, "Model-based safety validation of the automated driving function Highway Pilot," in 8th International Munich Chassis Symposium 2017, 2017, pp. 309-329.

[15] M. Pereira Cocron et al., "Evaluation Framework," ADAS\&ME Deliverable 7.1, Feb. 2018.

[16] European Commission, "CARE database - Statistics - accidents data Mobility and transport," Mobility and transport. [Online]. Available: /transport/road_safety/specialist/statistics_en. [Accessed: 10-Apr2018].

[17] G. R. de Campos, A. H. Runarsson, F. Granum, P. Falcone, and K. Alenljung, "Collision avoidance at intersections: A probabilistic threat-assessment and decision-making system for safety interventions," in Proceedings of the 17th International IEEE Conference on Intelligent Transportation Systems (ITSC), 2014, pp. 649-654.

[18] E. Dabbour and S. Easa, "Proposed collision warning system for right-turning vehicles at two-way stop-controlled rural intersections," Transportation Research Part C: Emerging Technologies, vol. 42, pp. 121-131, May 2014.

[19] O. Aycard et al., "Intersection safety using lidar and stereo vision sensors," in IV'2011-IEEE Intelligent Vehicles Symposium, 2011, pp. 863-869.

[20] J. Misener et al., "Cooperative Intersection Collision Avoidance System (CICAS): Signalized Left Turn Assist and Traffic Signal Adaptation," University of California, Berkeley, Richmond, CA, Final Report for Task Order 6608 UCB-ITS-PRR-2010-20, Mar. 2010.

[21] P. Nitsche, P. Thomas, R. Stuetz, and R. Welsh, "Pre-crash scenarios at road junctions: A clustering method for car crash data," Accident Analysis \& Prevention, vol. 107, pp. 137-151, Oct. 2017.

[22] Y. Kim, S. Tak, J. Kim, and H. Yeo, "Identifying major accident scenarios in intersection and evaluation of collision warning system," in 20th International Conference on Intelligent Transportation Systems (ITSC), Yokohama, Japan, 2017, pp. 1-6.

[23] M. D. McKay, R. J. Beckman, and W. J. Conover, "A Comparison of Three Methods for Selecting Values of Input Variables in the
Analysis of Output from a Computer Code," Technometrics, vol. 21, no. 2, pp. 239-245, May 1979.

[24] S. P. Olivares, N. Rebernik, A. Eichberger, and E. Stadlober, "Virtual Stochastic Testing of Advanced Driver Assistance Systems," in Advanced Microsystems for Automotive Applications 2015, Springer, Cham, 2016, pp. 25-35.

[25] M. Althoff and A. Mergel, "Comparison of Markov Chain Abstraction and Monte Carlo Simulation for the Safety Assessment of Autonomous Cars," IEEE Transactions on Intelligent Transportation Systems, vol. 12, no. 4, pp. 1237-1247, Dec. 2011.

[26] B. Canu, C. Perrin, J. Magnin, A. Canu, T. Serre, and T. Brenac, "Interest of in-depth investigation for studying the relation between speed and accident risk," presented at the 7th International Conference ESAR - Expert Symposium on Accident Research, Hannover, Germany, 2016, p. 11.

[27] W. E. Cliff and A. Moser, "Reconstruction of Twenty Staged Collisions with PC-Crash's Optimizer," in SAE 2001 World Congress, 2001.

[28] T. Helmer, Development of a Methodology for the Evaluation of Active Safety using the Example of Preventive Pedestrian Protection. Springer, 2014.

[29] U. Sander, "Opportunities and limitations for intersection collision intervention-A study of real world 'left turn across path' accidents," Accident Analysis \& Prevention, vol. 99, Part A, pp. 342-355, Feb. 2017.

[30] U. Sander and N. Lubbe, "Market penetration of intersection AEB: Characterizing avoided and residual straight crossing path accidents," Accident Analysis \& Prevention, vol. 115, pp. 178-188, Jun. 2018.

[31] J. Archer, "Indicators for traffic safety assessment and prediction and their application in micro-simulation modelling: a study of urban and suburban intersections," Dissertation, KTH Royal Institute of Technology, Stockholm, 2005.

[32] O. Bagdadi, "Estimation of the severity of safety critical events," Accident Analysis \& Prevention, vol. 50, pp. 167-174, Jan. 2013.

[33] T. De Ceunynck, "Defining and applying surrogate safety measures and behavioural indicators through site-based observations," Dissertation, Lund University, 2017.

[34] A. Laureshyn, Å. Svensson, and C. Hydén, "Evaluation of traffic safety, based on micro-level behavioural data: Theoretical framework and first implementation," Accident Analysis \& Prevention, vol. 42, no. 6, pp. 1637-1646, Nov. 2010.

[35] A. Laureshyn, T. De Ceunynck, C. Karlsson, Å. Svensson, and S. Daniels, "In search of the severity dimension of traffic events: Extended Delta-V as a traffic conflict indicator," Accident Analysis \& Prevention, vol. 98, pp. 46-56, Jan. 2017.

[36] S. M. S. Mahmud, L. Ferreira, M. S. Hoque, and A. Tavassoli, "Application of proximal surrogate indicators for safety evaluation: A review of recent developments and research needs," IATSS Research, vol. 41, no. 4, pp. 153-163, Dec. 2017.

[37] S. Kumar and D. Toshniwal, "Analysing road accident data using association rule mining," in 2015 International Conference on Computing, Communication and Security (ICCCS), 2015, pp. 1-6.

[38] L. Kaufman and P. J. Rousseeuw, Eds., Finding Groups in Data. Hoboken, NJ, USA: John Wiley \& Sons, Inc., 1990.

[39] P. J. Rousseeuw, "Silhouettes: A graphical aid to the interpretation and validation of cluster analysis," Journal of Computational and Applied Mathematics, vol. 20, pp. 53-65, Nov. 1987.

[40] R. Agrawal, T. Imielinski, and A. Swami, "Mining association rules between sets of items in large databases," Acm sigmod record, vol. 22, pp. 207-216, 1993.

[41] C. Hydèn, "The development of a method for traffic safety evaluation: The Swedish traffic conflicts technique,” Institut för Trafikteknik, LTH Lund, 1987.

[42] $\AA$. Svensson, "A method for analysing the traffic process in a safety perspective,” Dissertation, Lund University, Lund, Sweden, 1998.

[43] NHTSA, “Tire Pressure Monitoring System," FMVSS 138, Jul. 2001.

[44] R. Miller and Q. Huang, "An adaptive peer-to-peer collision warning system," in IEEE 55th Vehicular Technology Conference, 2002, pp. 317-321.

[45] A. Sobhani, W. Young, S. Bahrololoom, M. Sarvi, and others, "Calculating time-to-collision for analysing right turning behaviour at signalised intersections," Road \& Transport Research: A Journal of Australian and New Zealand Research and Practice, vol. 22, no. 3, p. 49, 2013. 